\title{
Uso de Estilos de Aprendizagem em Ambientes Educacionais Ubíquos
}

\author{
Rafael Araújo, UFU, rafael.araujo@ufu.br \\ Fabiano Dorça, UFU, fabianodor@ufu.br \\ Renan Cattelan, UFU, renan@ufu.br \\ Cíntia Santos, Escola Municipal de Educação Infantil Líria Emília Saraiva, \\ cnt.cintia@gmail.com
}

\section{Resumo}

As práticas pedagógicas têm sido constantemente melhoradas e alteradas com o auxílio de aparatos tecnológicos para alavancar o processo de ensino-aprendizagem. Com sistemas computacionais ubíquos e inteligentes, é possível criar mecanismos para que as individualidades dos estudantes possam ser levadas em consideração e, ainda, para permitir que os professores conheçam as características da turma e de seus alunos de forma mais rápida e dinâmica. Este artigo apresenta a experiência dos autores com um ambiente educacional que utiliza uma abordagem probabilística do modelo de Estilos de Aprendizagem (EAs) de Felder e Silverman para adaptar o conteúdo aos estudantes. Os resultados mostraram diferenças de EAs nas disciplinas analisadas e reforçam a ideia de que não são características fixas do indivíduo que está em constante transformação.

Palavras-chave: Ambientes Educacionais Ubíquos; Estilos de Aprendizagem; FSLSM.

\section{Using Learning Styles in Ubiquitous Learning Environments}

\begin{abstract}
Pedagogical practices have been constantly improved and modified with the help of technological gadgets to leverage the teaching-learning process. With intelligent computational systems, it is possible to create mechanisms to consider students' individualities and to allow teachers to get to know the characteristics of their classes and students more quickly and more dynamically. This article presents a case study of an educational system that uses a probabilistic approach of the Felder and Silverman Learning Styles (LS) Model to adapt the content to students. Results showed differences of LS in the analyzed courses and reinforce the idea that they are not fixed characteristics of individuals who are in constant transformation.
\end{abstract}

Keywords: Adaptive Educational Systems; Learning Styles; FSLSM.

\section{Introdução}

O surgimento de Ambientes Educacionais Ubíquos (AEUs) tem potencializado a geração automática de Objetos de Aprendizagem (OAs) (ARAÚJO et al., 2014). Entretanto, a simples criação de tais objetos não os tornam passíveis de serem personalizados. Assim, para que os sistemas computacionais consigam prover recursos personalizados, é importante que eles tenham informações suficientes tanto acerca das características de cada estudante quanto das características do conteúdo trabalhado. No caso do estudante, o mapeamento e armazenamento de suas características acontece por meio do chamado V. $17 \mathrm{~N}^{\circ}$ 3, dezembro, 2019 RENOTE DOI: 
Modelo do Estudante (ME), que captura e armazena tais informações. Por outro lado, as características do conteúdo, chamados de Objetos de Aprendizagem (OAs), são armazenadas por meio de metadados. Neste trabalho, o padrão de metadados IEEE-LOM (Learning Object Metadata) (IEEE, 2002) é utilizado, visto que é um padrão mundialmente utilizado nesse contexto.

Os sistemas computacionais têm se tornado cada vez mais inteligentes e dinâmicos a fim de criar experiências personalizadas aos seus usuários. Especialmente no contexto educacional, abordagens que visam melhorar a experiência de aprendizagem utilizando recursos de recuperação e personalização de conteúdo têm surgido para evitar a abordagem chamada de "one-size-fits-all”" (BRUSILOVSKY, 2001), ou seja, quando o mesmo conteúdo é apresentado da mesma maneira a todos os estudantes. Com isso, é possível criar abordagens automatizadas para que o conteúdo seja devidamente associado às características de cada estudante. Tais abordagens dão origem aos chamados Sistemas Educacionais Adaptativos (AKBULUT; CARDAK, 2012; BRUSILOVSKY, 2003). Um sistema educacional adaptativo considera as diferenças entre os estudantes e pode levar em consideração suas preferências, o conhecimento prévio, seus objetivos, seu contexto de aprendizagem, etc. Para que o conteúdo possa ser apresentado de forma individualizada, é necessário, então, que se conheça as características de cada um de seus usuários (por meio do $\mathrm{ME}$ ) bem como as características do conteúdo em si (representados na forma de OAs).

Em consonância com teorias pedagógicas consolidadas, como a de Piaget (PIAGET, 1983) em que o aluno é o protagonista na construção do seu conhecimento e tem formas discordantes de aprendizagem é que surge a necessidade de repensar as estratégias metodológicas e didáticas a fim de atender alunos com diferentes estruturas cognitivas de forma a manter seu interesse e possibilitar novas formas de aprendizagem. Dessa forma, a criação de ambientes inteligentes de aprendizagem tem o potencial de proporcionar uma melhor visão dos perfis de alunos como também dos recursos que podem ser utilizados para melhoria da aprendizagem.

Uma das possíveis abordagens, entre diversas, que podem ser consideradas para o propósito apresentado é a utilização de Estilos de Aprendizagem (EAs), que é um aspecto cognitivo fundamentado em teorias pedagógicas que tem sido estudado nesse contexto. Vários trabalhos apontam que sua utilização gera impactos positivos nos resultados de aprendizagem (ALSHAMMARI; ANANE; HENDLEY, 2015; EL-BISHOUTY et al., 2014). Em contrapartida, também existem trabalhos que questionam sua eficácia (AN; CARR, 2017; KIRSCHNER, 2017). No entanto, as críticas geralmente acontecem pelo fato de que, em geral, os EAs possuem classificações em extremidades antagônicas e os estudantes são classificados em uma delas, ou seja, sem considerar incertezas ou mesmo a mistura delas. Além disso, os instrumentos utilizados para se aferir EAs são muito extensos e cansativos, o que desmotiva os estudantes e faz com que eles desistam de respondê-los ou respondam sem a devida atenção.

Este trabalho tem como objetivo principal apresentar a experiência dos autores na construção e utilização de um ambiente educacional ubíquo baseado em estilos de aprendizagem em cenários reais de uma universidade brasileira. Tal sistema utiliza uma proposta probabilística do modelo de Felder e Silverman (FSLSM) (FELDER; SILVERMAN, 1988) com a inclusão de uma abordagem semiautomática para aferir os EAs. À medida que o estudante é avaliado, inferências sobre suas possíveis preferências de aprendizagem são realizadas e o modelo probabilístico é atualizado. Esse estudo visa explorar correlações dos estilos de aprendizagem dos estudantes com seu conhecimento prévio nas disciplinas e as interações realizadas no ambiente online. 
O restante deste artigo está organizado da seguinte maneira: inicialmente, o referencial teórico relacionado a esta pesquisa é apresentado na Seção 2; o método de pesquisa e a abordagem utilizada neste trabalho são descritos na Seção 3; os resultados obtidos e discussões acerca do estudo realizado são apresentados na Seção 4; e, por fim, as considerações finais são apresentadas na Seção 5.

\section{Referencial Teórico}

Esta seção apresenta os principais conceitos necessários para o entendimento da proposta ora apresentada.

\subsection{Ambientes Educacionais Ubíquos}

Mark Weiser (WEISER, 1991) definiu o conceito de Computação Ubíqua no final da década de 1980 para descrever a integração cada vez mais onipresente de dispositivos computacionais no cotidiano das pessoas. Uma das subáreas de pesquisa, chamada de Captura \& Acesso (C\&A), cria aplicações que gravam atividades diárias para futuras revisões. Especificamente no contexto educacional, o uso de tecnologia pode ajudar professores e estudantes no processo de ensino e aprendizagem por meio da automação de tarefas pedagógicas que podem ser acessadas em diferentes contextos, o que dá origem aos chamados Ambientes Educacionais Ubíquos (AEUs).

Salas de aulas equipadas com dispositivos computacionais, como projetores, lousas eletrônicas, microfones e câmeras de vídeo, produzem, de forma não intrusiva, artefatos multimídia capazes de simular experiências vividas em sala de aula. Aplicações de C\&A no contexto educacional permitem que professor prepare o conteúdo a ser ministrado. Então, diferentes mídias podem ser capturadas e geradas in loco no momento em que a aula é ministrada para que sejam posteriormente disponibilizadas aos estudantes para estudo. Além disso, professores e estudantes podem enriquecer o material disponibilizado com informações complementares no momento do acesso ao conteúdo (PIMENTEL et al., 2001).

Ambientes dessa natureza, ou seja, ambientes enriquecidos por tecnologia, são potenciais produtores de OAs, oferecem suporte para a geração automática de materiais educacionais e, ainda, são capazes de criar metadados de forma automática ou semiautomática que se tornam fonte de informação para auxiliar o processo de personalização e recomendação de conteúdo de acordo com as necessidades e preferências de cada estudante.

\subsection{Teoria de Estilos de Aprendizagem}

Estudos da literatura indicam que estudantes aprendem de maneira diferente uns dos outros (ESSALMI et al., 2015; GRAF et al., 2014). Estratégias de ensino que consideram as diferenças individuais dos estudantes têm sido estudadas há algum tempo, como é o caso de modelos relacionados com Estilos de Aprendizagem (EAs).

Um modelo de EA classifica os estudantes de acordo com a forma com que eles percebem e processam as informações recebidas em contextos educacionais. Tais modelos podem determinar como cada indivíduo interage e reage em um ambiente de aprendizagem, refletindo suas reais preferências(FELDER; SILVERMAN, 1988). Existem vários modelos de EA que descrevem aspectos distintos sobre como os estudantes preferem aprender.

No modelo proposto por Felder e Silverman (FSLSM), cada estudante pode ser classificado em oito EAs diferentes agrupados em quatro dimensões: Ativo/Reflexivo, Sensitivo/Intuitivo, Visual/Verbal e Sequencial/Global. Neste trabalho, o modelo FSLSM V. $17 \mathrm{~N}^{\circ}$ 3, dezembro, 2019 RENOTE DOI: 
foi escolhido, visto que ele é amplamente utilizado pela comunidade científica e se baseia na ideia de que cada estudante tem uma preferência (ou tendência) para um dos dois EAs de cada uma das quatro dimensões, o que representa uma característica diferencial desse modelo. Esta característica pode permitir que sistemas educacionais inteligentes criem MEs mais detalhados para proporcionar uma personalização mais precisa e experiências adaptativas mais efetivas.

As dimensões do modelo FSLSM e seus EAs incorporam características individuais relacionadas com organização, entendimento, processamento e transmissão de informação durante o processo de aprendizagem. Brevemente, cada dimensão pode ser descrita como:

- Percepção: classifica os estudantes de acordo com a forma com que eles percebem o conteúdo. Estudantes sensitivos preferem conteúdo mais concreto, como fatos e exemplos, enquanto estudantes intuitivos preferem conteúdo mais abstrato, como teorias e modelos matemáticos;

- Entrada: indica a forma com que os estudantes preferem receber o conteúdo. Estudantes visuais preferem recursos visuais enquanto estudantes verbais preferem recursos textuais ou falados;

- Processamento: classifica os estudantes em indivíduos que preferem atividades mais práticas e trabalhos em grupo em seu processo aprendizagem, classificados como estudantes ativos, ou indivíduos que exercem uma postura mais passiva, classificados como estudantes reflexivos;

- Organização: indica a forma com que os estudantes preferem que o conteúdo seja organizado. Estudantes sequenciais preferem que a apresentação seja feita de forma progressiva e sequencial enquanto estudantes globais preferem obter uma visão geral do que é ensinado, permitindo que sua navegação seja mais livre.

Geralmente, instrumentos manuais para aferir o EA de cada estudante são utilizados. O Index of Learning Styles (ILS) é um questionário composto por 44 questões utilizadas para identificar o EA de estudantes de acordo com o FSLSM. Cada uma das quatro dimensões contém 11 questões com duas alternativas cada. Ao final, os estudantes são classificados por valores de -11 a +11 em cada dimensão. Apesar de ser um instrumento validado estatisticamente (FELDER; SPURLIN, 2005), seu preenchimento manual causa descontentamento e desmotivação por parte dos estudantes, o que pode levar a eventuais respostas imprecisas.

\section{Material e Métodos}

Para a realização desta pesquisa, utilizou-se a plataforma educacional ubíqua Classroom eXperience (CX) (ARAÚJO et al., 2013, 2018), que conta com funcionalidades colaborativas, sociais, de personalização de conteúdo e gamificação. Por meio desta plataforma, professores preparam suas aulas para serem ministradas presencialmente em salas de aula, onde elas são gravadas com o auxílio de dispositivos computacionais, como lousa eletrônica, projetores, microfones e câmeras de vídeo. As aulas capturadas são disponibilizadas aos estudantes via Web. Os OAs gerados no CX são automaticamente relacionados com metadados armazenados no padrão IEEE-LOM (Learning Object Metadata) (IEEE, 2002). Além disso, os professores podem criar questões de múltiplaescolha (quizzes) relacionados à cada um dos slides preparados para que os estudantes avaliem seu conhecimento.

Dado que um EA é uma informação relacionada à aprendizagem dos indivíduos e ela pode ser utilizada como parâmetro de preferência individual para personalização de conteúdo, no presente trabalho foi empregada uma abordagem probabilística para 
armazenamento dos EAs do modelo FSLSM proposta por (DORÇA et al., 2013). Tal abordagem considera incertezas na classificação dos estudantes nas dimensões do modelo de EAs, sendo que cada uma das quatro dimensões do modelo FSLSM soma uma probabilidade de preferência de $100 \%$ para a dimensão, ou seja, a soma da probabilidade de preferência de cada um dos dois EAs da dimensão é igual a 1.

Ao todo, oito valores decimais são armazenados em pares - um par para cada dimensão do modelo. Cada EA é complementar ao seu respectivo par na mesma dimensão, por exemplo: como os EAs Ativo e Reflexivo fazem parte da dimensão Processamento, a soma dos valores da probabilidade de preferência ao EA Ativo e da probabilidade de preferência ao EA Reflexivo não pode exceder o valor 1. Esta representação dá uma natureza probabilística aos EAs, o que significa que o EA de cada estudante não é uma escolha única e nem mesmo é fixa. Significa, portanto, que os estudantes tendem a preferir um ou mais EAs com uma certa probabilidade e que esta preferência pode evoluir ao longo do tempo. A Tabela 1 mostra um exemplo da informação de EA armazenada de um estudante fictício com a distribuição de probabilidade para cada uma das dimensões do modelo.

Tabela 1 - Exemplo da informação de EA de um estudante fictício.

\begin{tabular}{|c|c|c|c|c|c|c|c|}
\hline \multicolumn{2}{|c|}{ Processamento } & \multicolumn{2}{c|}{ Percepção } & \multicolumn{2}{c|}{ Entrada } & \multicolumn{2}{c|}{ Organização } \\
\hline Ativo & Reflexivo & Sensitivo & Intuitivo & Visual & Verbal & Sequencial & Global \\
\hline 0,78 & 0,22 & 0,37 & 0,63 & 0,55 & 0,45 & 0,50 & 0,50 \\
\hline
\end{tabular}

Além disso, esta proposta faz a inicialização do modelo a frio, com $50 \%$ de adequabilidade para cada EA. Para evitar que os estudantes se desmotivem e respondam ao ILS com informações imprecisas, o questionário é dividido em sessões de login no sistema ao longo do semestre para que o processo de aferição de EAs se torne menos cansativo. Ao habilitar esta funcionalidade, os estudantes matriculados na disciplina recebem um texto informativo sobre o ILS que é apresentado em seu primeiro login, como mostrado na Figura 1(a). Em seguida, quatro perguntas - uma de cada dimensão do modelo - são apresentadas aos estudantes por meio de um ícone de notifícação identificado com a cor vermelha em cada nova sessão de login. A Figura 1(b) mostra um exemplo de quatro perguntas do questionário apresentadas aos estudantes.

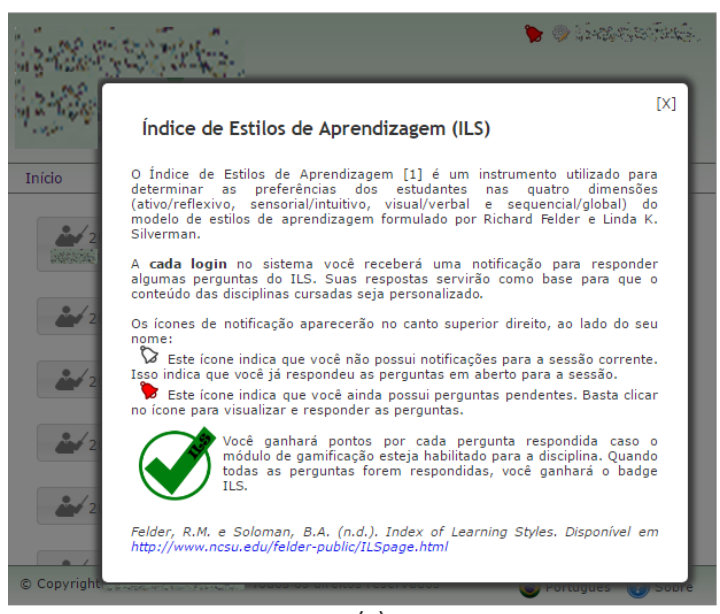

(a)

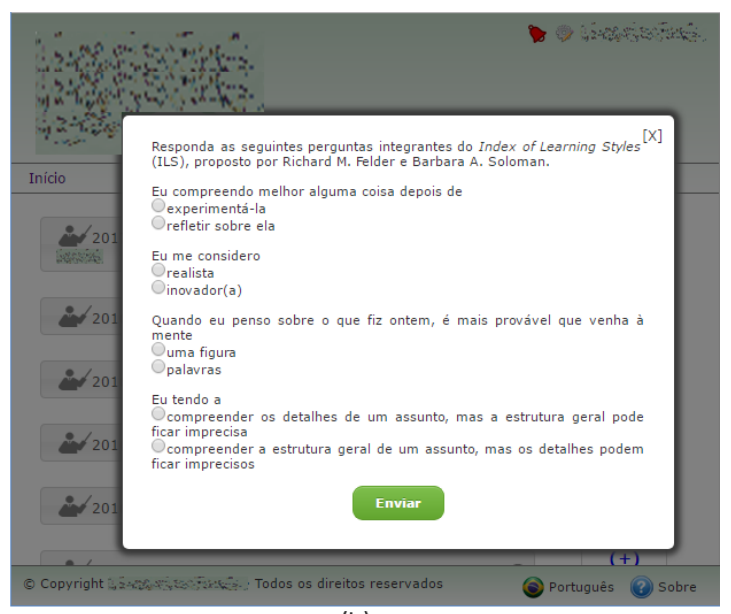

(b)

Figura 1 - (a) Caixa de diálogo com texto explicativo; (b) Quatro perguntas do ILS. 
Ao responder uma questão, a probabilidade de preferência ao EA correspondente a alternativa escolhida é incrementada em 4,54\% (50\% dividido por 11 questões de cada dimensão) e seu EA complementar é atualizado para o valor restante para atingir o valor 1. Ainda visando incentivar que os estudantes respondam às perguntas do questionário, pontuações são contabilizadas em um módulo de gamificação à medida que as perguntas são respondidas e, ao final de todas as perguntas, uma medalha (badge) referente ao questionário ILS é concedida ao estudante.

A abordagem proposta foi implementada no Modelo de Estudante da plataforma CX, que foi utilizada durante um semestre letivo por estudantes de cursos de graduação em Ciência da Computação e Sistemas de Informação de uma universidade pública brasileira. Ao todo, 43 estudantes das disciplinas de Interação Humano-Computador (IHC) e Arquitetura e Organização de Computadores (AOC) foram avaliados. Para esta finalidade, inicialmente aplicou-se um teste para se analisar o conhecimento prévio dos estudantes acerca de cada temática. Os testes foram compostos por dez questões de múltipla escolha e elaborados pelos professores de cada disciplina. A cada sessão de estudo online, o estudante poderia responder questões do ILS.

Para os dados que cada aluno obteve no teste de conhecimento prévio e suas respectivas pontuações nas dimensões dos EAs, aplicou-se o teste de Shapiro-Wilk para analisar se as amostras seguiam a distribuição normal. Devido à maioria das amostras não apresentar normalidade de resíduos, optou-se por verificar a existência de correlações entre as notas obtidas pelos estudantes e os EAs aferidos empregando-se a correlação de Spearman. Em seguida, dentro de cada uma dessas subamostras, empregou-se novamente a correlação de Spearman mas com o intuito de buscar correlações com os tipos de interações realizadas pelos estudantes. Neste estudo, dois tipos foram analisados: (i) resposta aos quizzes, indicando o número total de tentativas de respostas às questões de múltipla escolha cadastradas pelos professores, e (ii) atividades colaborativas, indicando a quantidade total de interações realizadas nas atividades de classificação de relevância de slides e classificação do tipo de recurso de aprendizagem de cada slide. Os professores podiam criar quantos quizzes achassem necessário e cada quiz podia ser respondido quantas vezes o aluno quisesse. As interações realizadas para classificar a relevância dos slides e indicar o tipo de recurso de aprendizagem foram consideradas "atividades colaborativas" nesse contexto, visto que são atividades que os usuários realizam com objetivo de refinar colaborativamente os metadados dos OAs.

O tipo de cada atividade e a quantidade realizada no âmbito da aprendizagem pode ser um bom indicativo de preferência do estudante. Por exemplo, atividades práticas e atividades colaborativas possuem uma natureza mais ativa que simplesmente uma leitura de slides, sem nenhuma interação além de "avançar" ou "voltar" no conteúdo, cuja natureza é mais passiva e pode-se ter uma noção de linearidade. Além disso, os quizzes representam um tipo de atividade que envolve dados e experimentação e leva a uma preferência mais sensitiva.

Os coeficientes de correlação resultantes foram analisados fazendo-se uso dos adjetivos de Davis (DAVIS, 1971), de modo que $r=1$ indica uma correlação perfeita; $0,70<r<0,99$ representa uma correlação muito alta; 0,50<r<0,69 demonstra uma correlação substancial; $0,30<r<0,49$ indica uma correlação moderada; $0,10<r<0,29$ demonstra uma correlação baixa e $0,01<r<0,09$ refere-se a uma correlação de caráter desprezível. Uma correlação negativa está vinculada ao lado negativo da escala do ILS (ou EA do lado esquerdo) e uma correlação positiva está relacionada com o lado positivo da escala do ILS (ou EA do lado direito). 


\section{Resultados e Discussões}

Em um primeiro momento, analisou-se a correlação dos EAs dos estudantes que participaram desta pesquisa em relação ao teste de conhecimento prévio realizado antes do início do semestre. A mediana geral do pré-teste dos 43 estudantes das duas disciplinas foi de 2 pontos. Assim, dois grupos foram criados, um com estudantes que obtiveram pontuação menor ou igual à mediana e o segundo com os estudantes que obtiveram pontuação maior que a mediana geral. A Tabela 2 mostra as correlações encontradas e a sua classificação de acordo com as categorias apresentadas na seção anterior.

Tabela 2 - Coeficientes da correlação de Spearman entre as pontuações obtidas pelos estudantes nos testes de conhecimento prévio e seus respectivos EAs.

\begin{tabular}{|c|c|c|c|c|c|}
\hline \multirow{2}{*}{ Grupo $^{a}$} & \multirow{2}{*}{$n^{b}$} & \multicolumn{4}{|c|}{ Estilos de Aprendizagem } \\
\hline & & Ati/Ref & Sen/Int & Vis/Ver & Seq/Glo \\
\hline BAIXO & 24 & $\begin{array}{c}-0,242 \\
(\text { moderada })\end{array}$ & $\begin{array}{c}0,322 \\
(\text { moderada })\end{array}$ & $\begin{array}{c}-0,083 \\
\text { (desprezivel) }\end{array}$ & $\begin{array}{c}0,414 \\
(\text { moderada })\end{array}$ \\
\hline ALTO & 19 & $\begin{array}{c}0,426 \\
(\text { moderada) }\end{array}$ & $\begin{array}{c}-0,339 \\
(\text { moderada })\end{array}$ & $\begin{array}{c}0,257 \\
(\text { baixa })\end{array}$ & $\begin{array}{c}-0,684 \\
\text { (substancial) }\end{array}$ \\
\hline
\end{tabular}

${ }^{a}$ Grupo BAIXO: Estudantes com pontuações no teste prévio menores ou iguais à mediana geral; Grupo ALTO: Estudantes com pontuações no teste prévio maiores que a mediana geral; ${ }^{\mathrm{b}} n$ : Tamanho das subamostras.

Os valores obtidos para os coeficientes da correlação de Spearman indicam que os estudantes do grupo com baixo conhecimento prévio apresentaram uma tendência para serem classificados em direção aos EAs Intuitivo (correlação moderada) e Global (correlação moderada). Segundo a definição do FSLSM, esses EAs indicam estudantes que preferem conteúdo abstrato, contendo uma visão geral, e que aprendem novos conceitos rapidamente, mas podem ser descuidados. Houve, também, uma correlação baixa com o EA Ativo, indicando uma tendência ao gosto de trabalhar em grupo.

Por outro lado, no grupo dos estudantes classificados com alto conhecimento prévio sobre o assunto, encontrou-se correlações moderadas para os EAs Reflexivo e Sensitivo e uma correlação substancial para o EA Sequencial, o que indica preferências para fatos e conteúdo concretos juntamente com um processo de raciocínio linear, o que indica que podem ser mais lentos na aquisição de conhecimento, porém são mais cuidadosos. De acordo com o FSLSM, o EA Reflexivo está relacionado com estudantes mais individualistas e que preferem uma aprendizagem por meio de observação e introspecção.

Em muitas disciplinas da área de computação, existe uma tendência de se utilizar uma abordagem top-down para exposição do conteúdo, ou seja, um modelo complexo é apresentado antes de seus detalhes, com o intuito de mostrar seus objetivos finais. É possível imaginar, por exemplo, a disciplina de AOC, na qual um diagrama arquitetural de alto nível pode representar um conteúdo abstrato e, a medida em que cada componente é especializado e explicado, chega-se em tópicos mais concretos e palpáveis. Sendo assim, faz sentido imaginar que os EAs Intuitivo e Global caminhem juntos para estudantes do grupo de baixo conhecimento inicial, ou seja, quem teve pouco ou nenhum contato com o conteúdo tem a necessidade de ter uma visão geral do conteúdo e, então, descobrir novas possibilidades e relacionamentos sobre o que é apresentado. Em contrapartida, com um alto conhecimento prévio, onde detalhes e fatos concretos acerca do conteúdo já são conhecidos, as correlações apontam outra direção, ou seja, EAs Sensitivo, Reflexivo e Sequencial, o que indica uma preferência por experimentação, 
observação e passo-a-passo linearmente (visto que já possuem uma noção do caminho a ser percorrido e, por isso, são mais detalhistas).

Após a análise do nível de conhecimento inicial, buscou-se entender os tipos de interação em cada um desses grupos, separados por disciplina. Algumas correlações moderadas e substanciais foram encontradas em ambas subamostras das duas disciplinas. O resultado mais expressivo está relacionado com a disciplina de IHC, em que todas as correlações (tanto no grupo de baixo quanto no grupo de alto conhecimento prévio) da dimensão Vis/Ver apontaram para o EA Visual, inclusive com uma correlação muito alta no grupo de alto conhecimento inicial que responderam aos quizzes da disciplina ( $\rho=$ 0,768 ). Isso indica que os estudantes que mais participaram ativamente das atividades de quizzes e interações colaborativas são aqueles que foram classificados como Visuais, independente do grupo de conhecimento inicial. Acredita-se que este resultado esteja relacionado com as características da disciplina, visto que é uma disciplina que contempla bastante elementos visuais de interface de software e que chama atenção de estudantes com esse perfil.

A disciplina AOC apresentou outro resultado interessante. O grupo de estudantes com baixo conhecimento inicial apresentou uma tendência para o EA Ativo em ambos os tipos de interação, enquanto o grupo de alto conhecimento inicial apresentou uma tendência oposta, apontando para o EA Reflexivo. Isso pode ser um indício de que estudantes que não conhecem o assunto buscam trabalhar em grupo para construir $o$ conhecimento (característica do perfil Ativo) e utilizam os quizzes como verificação, já os estudantes com conhecimento acerca do assunto têm mais confiança para classificar o conteúdo de acordo com sua relevância.

Tais resultados apontam para diferenças de EAs em se tratando de conhecimento prévio da disciplina. Além disso, as características intrínsecas de cada disciplina também podem exercer influência na forma com que os estudantes preferem ou se sentem mais à vontade com determinados conteúdos, ou seja, reforçando a ideia de que EAs não são características fixas do indivíduo, mas sim características voláteis que se adaptam de acordo com o contexto.

Ademais, a identificação do EA de cada estudante pode contribuir para a prática docente, sendo um recurso que permite que professores conheçam melhor seus estudantes de forma mais rápida e dinâmica e consigam acompanhar a evolução de tais características durante o processo de aprendizagem, tornando o sistema inteligente um facilitador do processo ensino-aprendizagem ao abordar de diferentes formas um mesmo conteúdo. Dessa forma, a questão pedagógica que envolve a constante transformação no processo ensino-aprendizagem pode ser aperfeiçoada ao longo do processo. Do ponto de vista dos estudantes, novas formas de aprendizagem poderiam ser experimentadas a fim de contribuir e facilitar a compreensão do conteúdo.

\section{Considerações Finais}

$\mathrm{O}$ fato de que as pessoas se comportam e aprendem em ritmos diferentes requer que as diferenças individuais sejam consideradas no processo de ensino e aprendizagem. E são com metodologias que consideram o aluno como parte da construção do seu processo de aprendizagem que traz a necessidade de motivar seu interesse pelo conhecimento que busca a partir de recursos que mantenham seu interesse e facilitem a aquisição do conhecimento. Uma das possíveis formas, entre diversas existentes para este propósito, é a utilização de EAs. Vários trabalhos apontam que a utilização de EAs gera impactos positivos nos resultados de aprendizagem. Por outro lado, também existem trabalhos que questionam sua eficácia, principalmente pelo fato de os modelos propostos não 
considerarem incertezas e, pelo contrário, classificarem os estudantes exclusivamente em uma categoria.

Sendo assim, este trabalho apresentou um a experiência dos autores com a construção e utilização de um AEU baseado em uma abordagem probabilística do modelo FSLSM. Resultados mostram que grupos de estudantes com baixo conhecimento inicial nas disciplinas possuem uma correlação moderada com os EAs Intuitivo e Global, o que pode estar relacionado com as características das disciplinas. Estudantes com alto conhecimento inicial apresentaram correlações com EAs em sentidos opostos, ou seja, Reflexivo (correlação moderada) e Sequencial (correlação substancial). Além disso, tipos de interação com o sistema em cada um dos grupos também podem indicar diferentes EAs em diferentes disciplinas, o que reforça a ideia de que EAs não são características fixas do indivíduo e que ao longo de seu processo de aprendizagem estas características podem ser modificadas.

De todo modo, estes resultados não indicam que os EAs estão relacionados somente com o parâmetro de expertise, mas que esta informação pode ser utilizada para efetuar ajustes de inicialização do modelo em trabalhos futuros. Além disso, pretende-se incluir uma abordagem baseada em padrões de interação no ambiente online para também ajustar as informações de EA do estudante.

Algumas limitações da abordagem podem ser apontadas, como a variabilidade de tipos de OA, visto que a estratégia de adaptação de conteúdo depende da existência de diferentes tipos de recurso para um mesmo conteúdo. Além disso, o tamanho amostral e outras disciplinas de outros domínios do conhecimento, além daquelas aqui apresentadas, podem resultar em diferentes conclusões.

\section{Agradecimentos}

Os autores agradecem o apoio das agências de pesquisa e instituições: CNPq, FAPEMIG, Coordenação de Aperfeiçoamento de Pessoal de Nível Superior - Brasil (CAPES) Código de Financiamento 001, FACOM/PROPP/UFU e PET/MEC/SESu.

\section{Referências}

AKBULUT, Y.; CARDAK, C. S. Adaptive educational hypermedia accommodating learning styles: A content analysis of publications from 2000 to 2011. Computers \& Education, Elsevier Science Ltd., Oxford, UK, v. 58, n. 2, p. 835-842, 2012.

ALSHAMMARI, M.; ANANE, R.; HENDLEY, R. J. The impact of learning style adaptivity in teaching computer security. In: Proceedings of the 2015 ACM Conference on Innovation and Technology in Computer Science Education. Vilnius, Lithuania: ACM, 2015. (ITiCSE'15), p. $135-140$.

AN, D.; CARR, M. Learning styles theory fails to explain learning and achievement: Recommendations for alternative approaches. Personality and Individual Differences, Elsevier Science Ltd., v. 116, p. 410-416, 2017.

ARAÚJO, R. D. et al. Personalization of interactive digital media in ubiquitous educational environments. In: Proceedings of the IEEE International Conference on Systems, Man and Cybernetics. Manchester, UK: IEEE, 2013. (SMC'13), p. 3955-3960.

ARAÚJO, R. D. et al. Autoria automática de objetos de aprendizagem a partir de captura multimídia e associação a estilos de aprendizagem. In: Anais do XXV Simpósio Brasileiro de Informática na Educação. Dourados, MS, BR: SBC, 2014. (SBIE'14), p. 229-238.

ARAÚJO, R. D. et al. A hybrid architecture for adaptive, intelligent and ubiquitous educational systems. In: ZHENG, R. (Ed.). Digital Technologies and Instructional Design for Personalized 
Learning. [S.1.]: IGI Global, 2018. p. 120-144.

BRUSILOVSKY, P. Adaptive hypermedia. User Modeling and User Adapted Interaction, Springer, v. 11, n. 1, p. 87-110, 2001.

BRUSILOVSKY, P. Developing Adaptive Educational Hypermedia Systems: From Design Models to Authoring Tools. In: MURRAY, T.; BLESSING, S. B.; AINSWORTH, S. (Eds.). Authoring Tools for Advanced Technology Learning Environments: Toward Cost-Effective Adaptive, Interactive and Intelligent Educational Software. Dordrecht: Springer Netherlands, 2003. p. 377-409.

DAVIS, J. A. Elementary Survey Analysis. Englewood Cliffs, NJ: Prentice-Hall, 1971.

DORÇA, F. A. et al. Comparing strategies for modeling students learning styles through reinforcement learning in adaptive and intelligent educational systems: An experimental analysis.

Expert Systems with Applications, v. 40, n. 6, p. 2092-2101, 2013.

EL-BISHOUTY, M. M. et al. Smart e-course recommender based on learning styles. Journal of Computers in Education, v. 1, n. 1, p. 99-111, 2014.

ESSALMI, F. et al. Generalized metrics for the analysis of E-learning personalization strategies. Computers in Human Behavior, v. 48, p. 310-322, 2015.

FELDER, R. M.; SILVERMAN, L. K. Learning and Teaching Styles in Engineering Education. Journal of Engineering Education, v. 78, n. 7, p. 674-681, 1988.

FELDER, R. M.; SPURLIN, J. Applications, Reliability and Validity of the Index of Learning Styles. International Journal of Engineering Education, v. 21, n. 1, p. 103-112, 2005.

GRAF, S. et al. Investigating the Effectiveness of an Advanced Adaptive Mechanism for Considering Learning Styles in Learning Management Systems. In: Proceedings of the 2014 IEEE 14th International Conference on Advanced Learning Technologies. Athens, Greece: IEEE, 2014. (ICALT'14), p. 112-116.

IEEE. LTSC 1484.12.1, Draft Standard for Learning Object Metadata, 2002. Learning Technology Standards Committee of the IEEE. Disponível em: $<$ http://ltsc.ieee.org/wg12/files/LOM_1484_12_1_v1_Final_Draft.pdf $>$.

KIRSCHNER, P. A. Stop propagating the learning styles myth. Computers \& Education, v. 106, p. 166-171, 2017.

PIAGET, J. A Epistemologia genética; Sabedoria e ilusões da filosofia ; Problemas de psicologia genética. São Paulo: Abril Cultural, 1983.

PIMENTEL, M. da G. et al. Supporting Educational Activities through Dynamic Web Interfaces. Interacting with Computers, Special Issue on Interacting with the Active Web, v. 13, n. 3, p. 353-374, 2001.

WEISER, M. The computer for the 21st century. Scientific American, v. 265, n. 3, p. 94-104, 1991. 